\title{
Technical Note: The 80/20 Technique for Posterior Lumbar Interbody Fusion - A Combination of Open Decompression and Percutaneous Pedicle Screw Fixation
}

\author{
Ralph J Mobbs ${ }^{1,2,3}$, Praveenan Sivabalan ${ }^{2,3^{*}}$, Jane $\mathbf{L i}^{2,3}$ and Peter Wilson ${ }^{1,2}$ \\ ${ }^{1}$ Department of Neurosurgery, Prince of Wales Private Hospital, Australia \\ ${ }^{2}$ Neuro Spine Clinic, Randwick, New South Wales, Australia \\ ${ }^{3}$ University of New South Wales, Kensington, New South Wales, Australia
}

\begin{abstract}
Objective: To illustrate a hybrid technique that involves a combination of open decompression and Posterior Lumbar Interbody Fusion (PLIF) and percutaneously placed pedicle screws. This technique allows for PLIF via a midline incision and approach, and decompression without compromise in operative time and visualisation. Furthermore, this approach is proposed to reduce post-operative wound pain by: smaller midline incision, significantly reduced muscle trauma by not dissecting the paraspinal muscles off the facet joint complex, avoidance of a posterolateral fusion to facilitate limited lateral muscle dissection off the transverse processes.
\end{abstract}

Summary of background data: PLIF fusion rates are comparable to posterolateral fusion rates, as well as providing greater sagittal and coronal balance. There is positive evidence that degenerative spondylolisthesis with canal and/or foraminal stenosis requires stabilisation when decompressed via laminectomy.

Methods: Patients with Grade I-II spondylolisthesis at L4/5 with moderate - severe canal/foraminal stenosis undergo a midline PLIF at L4/5, with closure of the midline incision. Percutaneous pedicle screws are inserted, therefore minimising local muscle trauma, with reduction of the spondylolisthesis performed using the pedicle screw construct. Rods are inserted percutaneously to link the L4 and L5 pedicle screws. Image intensification is used to confirmed satisfactory screw placement and reduction of the spondylolisthesis.

Conclusion: Percutaneous lumbar pedicle screws can be combined with a standard midline PLIF to reduce postoperative wound pain while allowing for satisfactory screw placement.

Keywords: Percutaneous lumbar pedicle screws; Posterior lumbar interbody fusion; Spondylolisthesis; 80/20 technique; 50/50 technique

\section{Introduction}

Degenerative lumbar spondylolisthesis provides a challenging clinical entity. When associated with lumbar canal and/or foraminal stenosis the patient can present with claudicant and/or radicular symptoms respectively. Positive outcomes can be seen with operative intervention when compared to conservative management. The spondylolisthesis arm of the SPORT trial concluded that in a nonrandomised as-treated environment (with control of potentially confounding baseline factors), outcomes were significantly better in regards to pain and function after 2 years for patients with degenerative spondylolisthesis and spinal stenosis than those treated non-operatively (SPORT Trial) [1]. Its correlation with mechanical low back pain is less clear and will not be discussed here.

One of the treatment methods proposed for degenerative spondylolisthesis with claudicant and/or radicular symptoms is lumbar laminectomy with instrumented fusion. This can take the form of a posterior approach (pedicle screw fixation \pm posterolateral graft \pm posterior or transforaminal lumbar interbody fusion: PLIF/TLIF), as well as an anterior approach (anterior lumbar interbody fusion: ALIF), or a combination of the above. PLIF has been shown to be equivocal to TLIF in regards to good outcomes post-operatively [2]. PLIF has been shown in one prospective study to have a higher fusion rate than posterolateral fusion alone, but at the cost of a higher complication rate related to hardware biomechanics [3]. Kim et al. [4], found that in directly comparing PLIF, posterolateral fusion and PLIF with posterolateral fusion, that there was no difference in regards to clinical results and union rates between the three; however, they noted that PLIF alone allowed for less donor site pain, shorter operating time and less blood loss (it has been noted that these benefits are, at least in part, attributable to not taking any iliac crest bone graft for the PLIF group) [4]. In a review of PLIF versus posterolateral fusion for management of isthmic spondylolisthesis, a higher fusion rate of $93 \%$ for PLIF versus $68 \%$ for posterolateral fusion was found, but only with a statistically significant improvement of outcome for high grade slipping managed with PLIF; there was no difference in outcomes for low grade slip [5]. An earlier review noted improved statistical parameters for PLIF over posterolateral fusion in isthmic spondylolisthesis, but without any clinical outcome variation [6].

Disadvantages of an open posterior approach include the morbidity of increased muscle dissection, increased post-operative wound pain (both short and long term), slower mobilisation post-operatively (and subsequent longer hospital stay), as well as increased intra-operative blood loss. An alternative method to the "traditional" open approach is to provide a minimally invasive method by use of percutaneous pedicle screws in combination with a minimally invasive bilateral laminotomy and PLIF. However, this method is compromised by longer operating times and higher complication rates [7].

*Corresponding author: Praveenan Sivabalan, Suite 3, Level 7, Prince of Wales Private Hospital, Randwick NSW 2031, Australia, Tel: +61-4-0196-5057; Fax: +61-29650-4943; E-mail: praveenan_sivabalan@hotmail.com

Received February 02, 2012; Accepted May 28, 2012; Published June 10, 2012

Citation: Mobbs RJ, Sivabalan P, Li J, Wilson P (2012) Technical Note: The 80/20 Technique for Posterior Lumbar Interbody Fusion - A Combination of Open Decompression and Percutaneous Pedicle Screw Fixation. J Spine 1:119. doi:10.4172/2165-7939.1000119

Copyright: $\odot 2012$ Mobbs RJ, et al. This is an open-access article distributed under the terms of the Creative Commons Attribution License, which permits unrestricted use, distribution, and reproduction in any medium, provided the original author and source are credited. 
An alternative method that is presented here involves a combination of the open and minimally invasive approaches, with the aim of maximising the benefits and avoiding the disadvantages of both the open and minimally invasive approaches. An open laminectomy and PLIF is performed at the pathological disc level to minimise operative time and to provide adequate exposure. This allows minimisation of intra-operative adverse events as well as provide a contingency in the event of adverse events should they occur (e.g. incidental durotomy). A PLIF alone is used and a posterolateral graft is excluded to minimise muscle dissection and retraction laterally, without compromising rates of fusion. Finally, percutaneous pedicle screws are placed in all pedicles to minimise muscle dissection off the facet joints to minimise postoperative wound pain (Figure 1).

\section{Technical Note}

The "80/20 Technique" (Figure 2) name was coined by the senior author (RJM) to describe the relative importance of each step in the procedure. The initial " $80 \%$ " is the primary goal of the technique: decompression of the neurological elements, preparation of the vertebral endplates and insertion of an interbody cage on either side of the thecal sac (Figure 3). The final " $20 \%$ " is the percutaneous insertion of the pedicle screws and reduction of the spondylolisthesis. The senior author has also previously described the "50/50 Technique" (Figure 4). In this case, the caudal pedicle screws are inserted via an open approach. This technique may be required if the caudal pedicle anatomy is difficult to determine using Anterior-Posterior X-ray and the surgeon is not comfortable with inserting percutaneous pedicle screws at that level.

\section{The $80 / 20$ Technique is as follows:}

Step 1: Under general anaesthesia, the patient is positioned prone on the Jackson table or similar operating table. It is essential to allow a radiolucent operative table at the level of the surgery to facilitate anteroposterior (AP) image intensifier x-rays.

Step 2: A midline incision is performed directly over the L4/5 disc space using X-ray to confirm the level of the spondylolisthesis. Lateral retraction is not necessary and therefore a short incision only is required. Most incisions are between $3.5-5 \mathrm{~cm}$. A retractor system of the surgeons' choice is used and a laminectomy at L4/5 is performed.

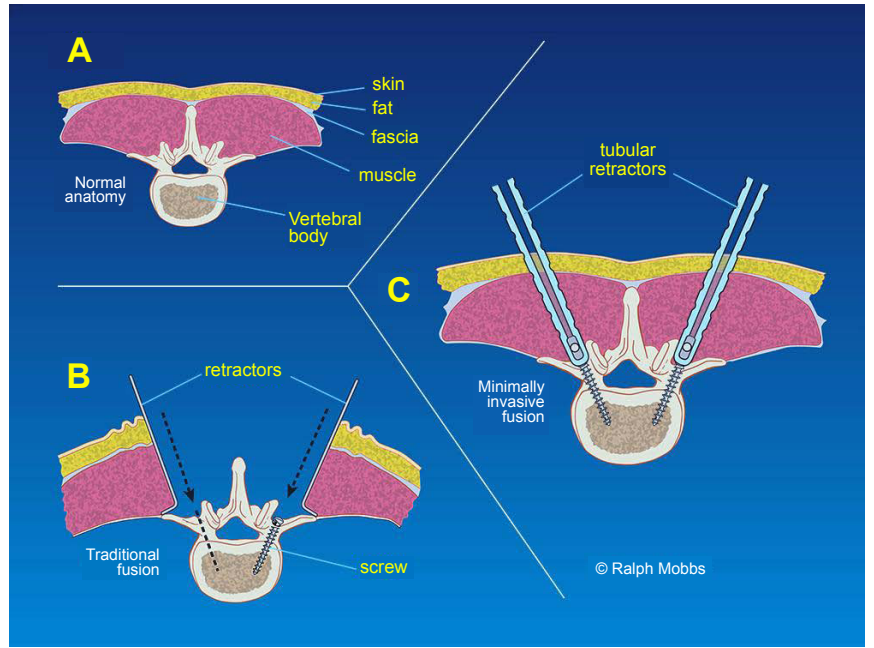

Figure 1: Rationale for MIS pedicle screw fixation: Minimization of muscle trauma with percutaneous pedicle screw insertion technique.

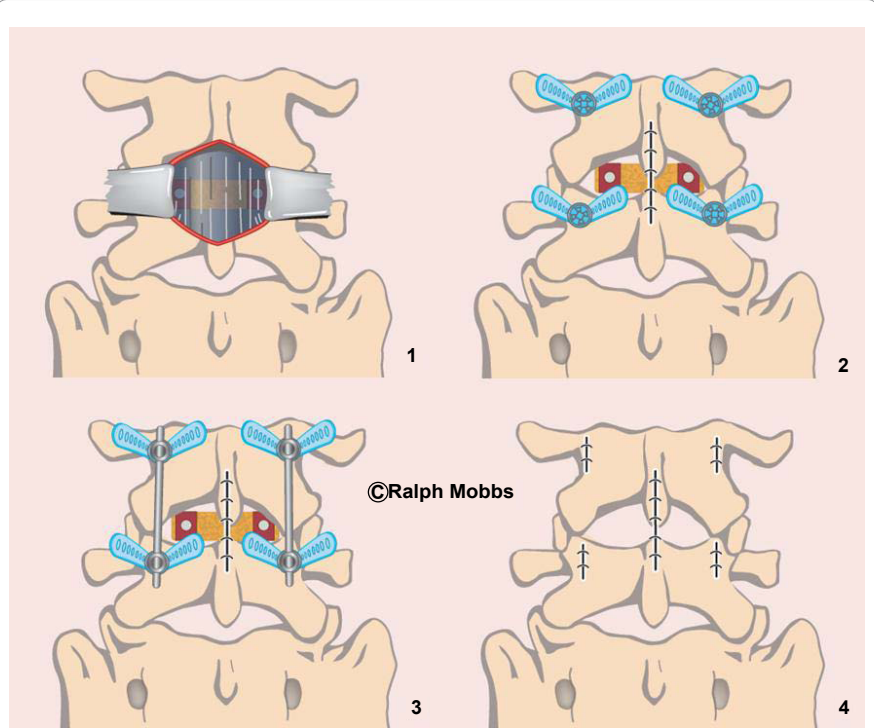

Figure 2: Workflow with $80 / 20$ technique: 1 . Midline incision and Posterior Lumbar Interbody Fusion performed. 2. Closure of the midline incision. 3. Percutaneous screw insertion via $x 4$ incisions with reduction using the pedicle screw construct. 4. Closure of the percutaneous incisions.

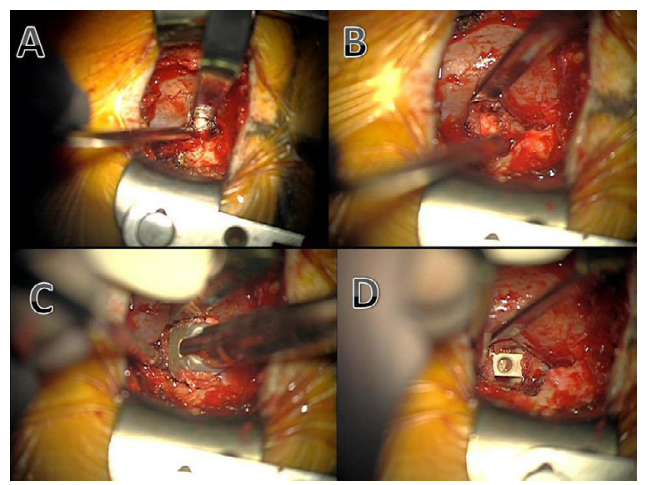

Figure 3: Midline PLIF Technique via Mini-open approach: A. Midline incision, decompression and preparation of interbody (disc) space. B. Endplate preparation. C. Insertion of rotatable cage packed with graft. D. Interbody cage in position.

A bilateral medial facetectomy at L4/5 with rhizolysis of both $\mathrm{L} 5$ nerve roots is undertaken. The disc at $\mathrm{L} 4 / 5$ is then removed and the endplates prepared (Figure 3 ). The bone from the $\mathrm{L} 4$ spinous process, laminae and L4/5 facets was cleaned of residual ligament/soft tissue and milled using a bone mill. It was then combined with osteobiologic material before being packed into two PLIF cages and inserted into the L4/5 disc space. After haemomstasis, the midline wound was closed in layers (Figure 2).

Step 3: The X-ray/II machine is moved into position to target the L4 and L5 pedicles. A Jamshidi needle is introduced via a stab incision along the lateral aspect of the pedicle on the AP view. The Jamshidi is introduced into the pedicle to a depth of $20-25 \mathrm{~mm}$ making sure not to breach the medial border of the pedicle wall on the AP view. Lateral $\mathrm{X}$-ray is performed to confirm the position of the Jamshidi into the vertebral body. After confirmation of the pedicles being penetrated by the needle, the trochar is removed and Kirschner $(\mathrm{K})$-wires introduced down the barrel of the Jamshidi needle. Their position is then confirmed prior to advancement of the K-wire through the pedicle under lateral 
fluoroscopy. Once a satisfactory penetration of the pedicle with the $\mathrm{K}$-wire was completed, the Jamshidi needle is removed whilst taking care to keep the K-wire in the same position. Appropriate skin incisions then need to be made. A pedicle tap is introduced down the K-wire, through the pedicle into the trabecular bone of the vertebral body and is confirmed with the image intensifier. The tap is then removed and appropriate pedicle screws (measurements based on pre-operative CT scans) were sited. Confirmation of pedicle screw placement is achieved with the image intensifier. Reduction of the spondylolisthesis is then performed using the instrumentation of the surgeon's choice (Figure 5 ). At the completion of the case, the 4 stab incisions are closed (Figure 2) with a single suture for the deep fascial and a single suture for the skin incision.

Step 4: Following reversal of anaesthesia, the patient is extubated post-operatively and transferred to the ward. Mobilisation can be attempted from day 1 post-op. Post-operative CT of the lumbar spine allows confirmation of reduction of the spondylolisthesis, as well as

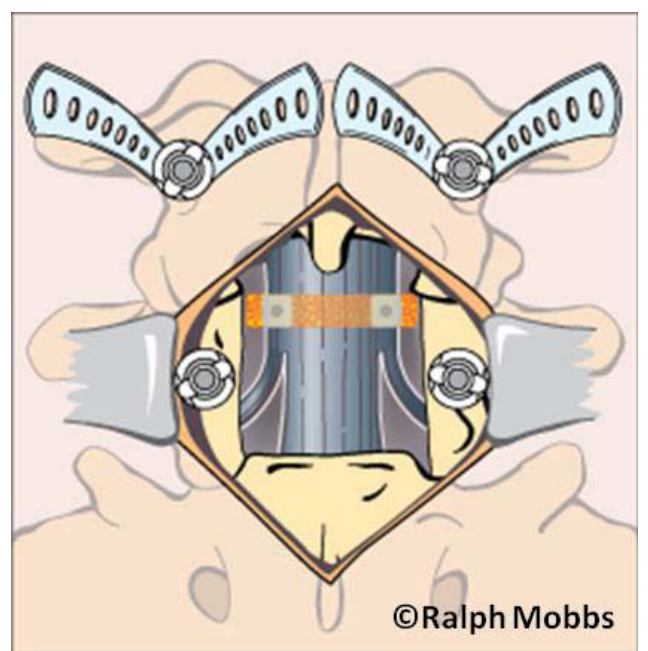

Figure 4: Workflow with 50/50 technique: Midline incision and Posterior Lumbar Interbody Fusion performed and insertion of pedicle screw into the caudal pedicle. Insertion of percutaneous screw into the cranial pedicle, therefore avoiding damage of the cranial/mobile facet joint.

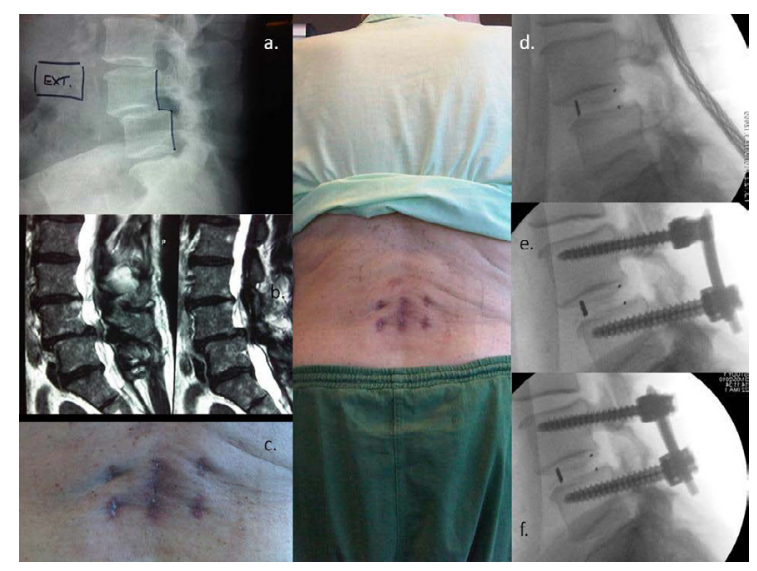

Figure 5: Pre and Post Operative Case example: A) L4/5 Spondylolisethesis. B) Severe canal stenosis. C) Final appearance of incisions at 4 weeks postop. D) Initial posterior lumbar interbody fusion performed. E) Insertion of percutaneous screws. F) Reduction manouver and correction of spondylolisethesis. satisfactory positioning of the interbody devices, bone graft and all four pedicle screws. Follow up is routinely performed at 6 weeks and 3 months with flexion/extension $\mathrm{x}$-rays views to confirm a solid fusion and reduction of the spondylolisthesis at $\mathrm{L} 4 / 5$.

\section{Clinical Results}

A clinical study was conducted from 2007 - 2011 to compare the prospective results of open versus minimally invasive fusion (80/20 technique) for degenerative lumbar spine pathologies. Eightytwo patients were studied (41 MIS spinal fusion, 41 open surgical equivalents) under a single surgeon (Senior author - RJM). Data collected on all patients included: Oswestry Disability Index (ODI), Short Form 12 (SF-12) v1, Visual Analogue Scale (VAS) and Patient Satisfaction Index (PSI), length of hospital stay, time to mobilise, postoperative medication and complications. Inclusion criteria consisted of patients aged 18-75 with degenerative pathologies only included. All patients complained of either back pain, radiculopathy, claudication or a combination of these three symptoms. All patients had pain resistant to prolonged (at least six months) conservative therapy.

The ODI and SF12 were utilised to analyse the impact of these surgical techniques on patient disability and quality of life, whilst the VAS assessed pain. Both groups showed significant improvements in quality of life and reduction in disability following their operations, with ODI falling from $54 \%$ to $22 \%$ for the MIS technique $(\mathrm{P}<0.0001)$ within the mean 16 month follow-up time period, and from $52 \%$ to $28 \%$ for the open technique $(\mathrm{P}<0.0001)$. Significant reductions in pain postoperatively were observed following each technique, with VAS falling from 7.9 to 2.4 for the MIS technique $(\mathrm{P}<0.0001)$ and from 8.2 to 3.3 for the open technique $(\mathrm{P}<0.0001)$. Postoperative pain was significantly lower following the MIS technique (2.4 vs. 3.3), but despite this, the amount of pain relief (VAS change) provided by both procedures were not significantly different.

A similar proportion of MIS (83.33\%) and open (78.57\%) patients were satisfied undertaking surgery for the benefit they received with their procedure. However surgery met the expectations of a significantly greater proportion of MIS patients than open patients $(\mathrm{P}=0.0236)$ The results for PSI are illustrated in Figure 6 . The minimally invasive technique resulted in significantly shorter hospital stay $(\mathrm{P}=0.0016)$ and time to mobilise $(\mathrm{P}=0.0021)$ after surgery than the open technique. The MIS group had a significantly lower postoperative opioid usage ( 85.90 vs. $168.9 \mathrm{mg}$ of IV morphine) than the open group ( $\mathrm{P}=0.0130)$. However the non-opioid usage between the MIS and open groups (26.9 vs. $30.84 \mathrm{~g}$ of oral paracetamol) was not significantly different.

The minimally invasive cohort was found to have significantly lower postoperative pain, and to have met the expectations of a significantly greater proportion of patients than conventional open surgery. The minimally invasive approach also had significantly shorter length of stay, time for mobilisation, lower opioid use and a reduced total complication rate. In our study minimally invasive techniques provide similar efficacy to the conventional open technique, and proves to be superior in regards to patient satisfaction, length of hospital stay, time to mobilise and complication rates.

\section{Discussion}

Posterior lumbar decompression and fusion is an evolving technique in an attempt to provide symptomatic and functional relief from a complex degenerative process. There are currently multiple alternatives to approach the pathology of degenerative spondylolisthesis which potentially creates a decision and management 


\section{PSI percentages}

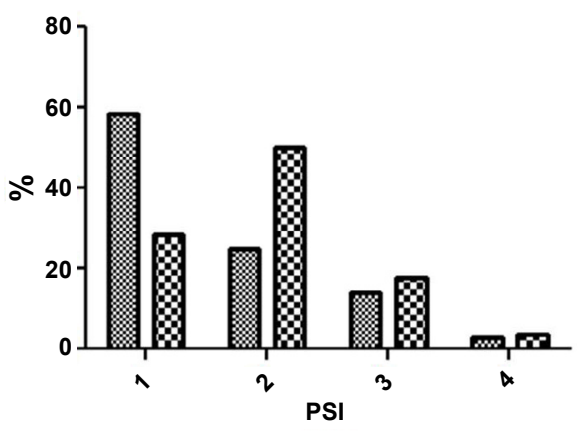

Figure 6: Patient Satisfaction Index. 1 - Surgery met my expectation, 2 - I did not improve as much as I had hoped but I would undergo the same operation for the same results, 3 - Surgery helped but I would not undergo the same operation for the same outcome, $4-\mathrm{I}$ am the same or worse as compared to before surgery.

dilemma. The SPORT trial, spondylolisthesis arm, concluded that operative management provides superior results when compared to non-operative management [1].

PLIF has been shown previously to provide high fusion rates that are at least equivocal, if not superior than postero-lateral fusion, with potential for correction of the spondylolisthesis and improvements in coronal and sagittal balance [8]. However, there is significant morbidity involved with regards to intra-operative blood loss, postoperative wound pain and delayed mobilisation post-operatively with the traditional 'open' approach. Minimally invasive pedicle screw fixation and minimally invasive TLIF using the METRx (Medtronic, Memphis, USA) system has been combined previously with the authors advocating: decreased blood loss, wound pain and average length of post-operative hospital stay. They do acknowledge, however, that the limited exposure does provide a potential environment for an increased chance of adverse events intra-operatively and a reduced operative field to correct any adverse event such as an unintended durotomy. Previous literature reveals that intra-operative durotomy rates are significantly increased, and the length of the procedure is also increased $[7,9,10]$. By providing an open laminectomy and PLIF procedure, the caveats of the minimally invasive TLIF/PLIF are avoided but with retention of the benefits of the percutaneous pedicle screws.

One potential source for increased post-operative wound pain is muscle dissection off the facet joints and transverse process. For a traditional open fusion, this is necessary to provide exposure for the pedicle screw entry points, especially the most rostral screw. Using percutaneous pedicle screws allows for minimal muscle dissection and avoidance of this morbidity.

Another potential source for increased post-operative wound pain is far lateral muscle dissection off the transverse processes to allow for a posterolateral graft. As this operation involves PLIF alone, no lateral dissection of muscle off the TP's is necessary.

Finally, in combination with the above two pain prevention strategies, the smaller exposure required both laterally and craniocaudally, allows for a more minimalistic incision that provides less soft tissue dissection, without compromising access. Wound size has previously shown to be independent of post-operative pain [11], but at the very least it is logical that a smaller wound facilitates reduced muscular exposure, and greater patient satisfaction.
The senior author (RJM) has performed 53 " $80 / 20$ " type procedures for degenerative spondylolisthesis. To date, no patient has required a blood transfusion with the average length of stay less than 3.7 days. In addition, over $50 \%$ of patients have not required morphine/narcotic based analgesia in the postoperative period.

\section{Conclusion}

The " $80 / 20$ " approach proposed by the authors has been successfully employed at our institution with encouraging results. The method of open PLIF and percutaneous pedicle screw fixation allows for minimisation of muscular dissection to reduce morbidity, reduce postoperative pain medication requirements and allow earlier mobilisation, whilst providing effective decompression and stabilisation of the degenerative motion segment.

\section{References}

1. Weinstein JN, Lurie JD, Tosteson TD, Hanscom B, Tosteson AN, et al (2007) Surgical versus nonsurgical treatment for lumbar degenerative spondylolisthesis. N Engl J Med 356: 2257-2270.

2. Yan DL, Pei FX, Li J, Soo CL (2008) Comparative study of PILF and TLIF treatment in adult degenerative spondylolisthesis. Eur Spine J 17: 1311-1316.

3. Cheng L, Nie L, Zhang L (2009) Posterior lumbar interbody fusion versus posterolateral fusion in spondylolisthesis: a prospective controlled study in the Han nationality. Int Orthop 33: 1043-1047.

4. Kim KT, Lee SH, Lee YH, Bae SC, Suk KS (2006) Clinical outcomes of 3 fusion methods through the posterior approach in the lumbar spine. Spine (Phila $\mathrm{Pa}$ 1976) 31: 1351-1357.

5. Dehoux E, Fourati E, Madi K, Reddy B, Segal P (2004) Posterolateral versus interbody fusion in isthmic spondylolisthesis: functional results in 52 cases with a minimum follow-up of 6 years. Acta Orthop Belg 70: 578-582.

6. La Rosa G, Conti A, Cacciola F, Cardali S, La Torre D, et al. (2003) Pedicle screw fixation for isthmic spondylolisthesis: does posterior lumbar interbody fusion improve outcome over posterolateral fusion? J Neurosurg 99: 143-150.

7. Park Y, Ha JW (2007) Comparison of one-level posterior lumbar interbody fusion performed with a minimally invasive approach or a traditional open approach. Spine (Phila Pa 1976) 32: 537-543.

8. Wang JC, Mummaneni PV, Haid RW (2005) Current treatment strategies fo the painful lumbar motion segment: posterolateral fusion versus interbody fusion. Spine (Phila Pa 1976) 30: S33-S43.

9. Foley KT, Holly LT, Schwender JD (2003) Minimally invasive lumbar fusion Spine (Phila Pa 1976) 28: S26-S35

10. Khoo LT, Palmer S, Laich DT, Fessler RG (2002) Minimally invasive percutaneous posterior lumbar interbody fusion. Neurosurgery 51: S166-S181.

11. Datta G, Gnanalingham KK, Peterson D, Mendoza N, O'Neill K, et al. (2004) Back pain and disability after lumbar laminectomy: is there a relationship to muscle retraction? Neurosurgery 54: 1413-1420. 